\title{
Pulmonary embolism: CT signs and cardiac biomarkers for predicting right ventricular dysfunction
}

\author{
T. Henzler*,\#,§, S. Roeger",\$, M. Meyer*,\#, U.J. Schoepf*, J.W. Nance Jr", D. Haghi", \\ W.E. Kaminski ${ }^{+}$, M. Neumaier ${ }^{+}$, S.O. Schoenberg* and C. Fink*
}

ABSTRACT: The aim of this study was to prospectively evaluate the accuracy of quantitative cardiac computed tomography (CT) parameters and two cardiac biomarkers ( $\mathrm{N}$-terminal-pro-brain natriuretic peptide (NT-pro-BNP) and troponin I), alone and in combination, for predicting right ventricular dysfunction (RVD) in patients with acute pulmonary embolism.

557 consecutive patients with suspected pulmonary embolism underwent pulmonary CT angiography. Patients with pulmonary embolism also underwent echocardiography and NT-proBNP/troponin I serum level measurements. Three different CT measurements were obtained (right ventricular (RV)/left ventricular (LV)axial, RV/LV4-CH and RV/LVvolume). CT measurements and NTpro-BNP/troponin I serum levels were correlated with RVD at echocardiography.

77 patients with RVD showed significantly higher RV/LV ratios and NT-pro-BNP/troponin I levels compared to those without RVD (RV/LVaxial $1.68 \pm 0.84$ versus $1.00 \pm 0.21$; RV/LV4-CH $1.52 \pm 0.45$ versus $1.01 \pm 0.21$; RV/LVvolume $1.97 \pm 0.53$ versus $1.07 \pm 0.52$; serum NT-pro-BNP $6,372 \pm 2,319$ versus $1,032 \pm 1,559 \mathrm{ng} \cdot \mathrm{L}^{-1}$; troponin I $0.18 \pm 0.41$ versus $0.06 \pm 0.18 \mathrm{~g} \cdot \mathrm{L}^{-1}$ ). The area under the curve for the detection of RVD of RV/LVaxial, RV/LV4-CH, RV/LVvolume, NT-pro-BNP and troponin I were $\mathbf{0 . 8 4}, 0.87,0.93,0.83$ and 0.70 respectively. The combination of biomarkers and $\mathrm{RV} / \mathrm{LV}$ volume increased the AUC to 0.95 (RV/LVvolume with NT-pro-BNP) and 0.93 (RV/LVvolume with troponin I).

$\mathrm{RV} / \mathrm{LV}$ volume is the most accurate CT parameter for identifying patients with RVD. A combination of RV/LVvolume with NT-pro-BNP or troponin I measurements improves the diagnostic accuracy of either test alone.

KEYWORDS: Cardiopulmonary inter-relationships, cardiopulmonary testing, cardiovascular, computed tomography, critical care medicine, pulmonary circulation

$\mathbf{R}$ ight ventricular dysfunction (RVD) is a predictor of poor outcome in patients with acute pulmonary embolism [1]. Thus, risk stratification relies on early detection of RVD in order to identify normotensive high-risk patients who might benefit from more aggressive therapies, such as thrombolysis or embolectomy $[2,3]$.

While echocardiography is considered the reference standard for assessing RVD in patients with acute pulmonary embolism [4-6], a multitude of recent studies have evaluated various morphometric parameters from pulmonary computed tomography (CT) angiography for predicting adverse outcomes or early death in patients with acute pulmonary embolism [7-13]. One of the most frequently investigated parameters is the ratio of right ventricular (RV) to left ventricular (LV) diameters as measured on transverse CT images or reconstructed four-chamber $(4-\mathrm{CH})$ views [7, 12]. Recent reports suggest that 3dimensional (3D) assessment of ventricular volumes is superior to diameter measurements for determining RVD in patients with pulmonary embolism [14-16]. However, studies that specifically compare the accuracy of CT signs for predicting RVD, as assessed by echocardiography, are limited and, to date, have not included ventricular volume measurements $[17,18]$.

Beyond imaging signs, cardiac biomarkers such as N-terminal pro-brain natriuretic peptide (NTpro-BNP) and troponin I have been proposed as predictors of clinical outcome in patients with acute pulmonary embolism [1, 19, 20]. NT-proBNP is secreted due to RV shear stress whereas increased levels of cardiac troponin I result from myocardial necrosis after severe RV pressure overload or a long duration of pressure overload that causes RV myocardial necrosis.
AFFILIATIONS

*Institutes of Clinical Radiology and Nuclear Medicine, and ${ }^{+}$Clinical Chemistry

"1st Dept of Medicine, University Medical Center Mannheim, Medical Faculty Mannheim - Heidelberg University, Mannheim, Germany. \#Dept of Radiology and Radiological Science, Medical University of South Carolina, Charleston, SC, USA.

${ }^{\text {s}}$ These authors contributed equally to this study.

CORRESPONDENCE

T. Henzler

Institute of Clinical Radiology and Nuclear Medicine, University Medical Center Mannheim

Medical Faculty Mannheim Heidelberg University Theodor-Kutzer-Ufer 1-3 D-68167 Mannheim Germany

E-mail: thomas.henzler@ medma.uni-heidelberg.de

Received:

May 252011

Accepted after revision:

Aug 162011

First published online:

Sept 292011 
Therefore, the aim of this study was to prospectively evaluate the accuracy of quantitative cardiac CT parameters, obtained from pulmonary CT angiography, and two cardiac biomarkers (NT-pro-BNP and troponin I), alone and in combination, for predicting RVD on echocardiography in patients with acute pulmonary embolism.

\section{MATERIALS AND METHODS Study population}

Our local ethics committees (Medical Faculty, Heidelberg University, Mannheim, Germany) approved this prospective study, and all patients gave written informed consent. Between August 2008 and June 2009, 575 consecutive patients with suspected pulmonary embolism underwent pulmonary CT angiography. Of those, 77 (13.4\%) had acute pulmonary embolism and were enrolled in the study. These included 42 males and 35 females with a mean age of $63 \pm 15.8$ yrs. Medical records of all patients were reviewed for the presence of congestive heart failure, cancer, myocardial infarction, chronic kidney disease, pulmonary hypertension and sepsis at the time of admission to CT and/or 2 weeks prior to admission.

\section{Echocardiographic assessment of RVD}

Echocardiography data were obtained with Vivid 7 and Vivid 1 (GE Healthcare, Chalfont St Giles, UK) ultrasound scanners. All examinations were performed within $24 \mathrm{~h}$ after the onset of symptoms by two cardiologists (S. Roeger and D. Haghi), who were blinded to NT-pro-BNP serum levels and CT measurements. The echocardiographic protocol included apical 2-, 3and $4-\mathrm{CH}$ views, parasternal long- and short-axis views and subcostal views. Digitised echocardiographic data were analysed by both investigators in a consensus reading using the EchoPAC PC (GE Medical Systems, Milwaukee, WI, USA) software package. Specifically, the right ventricle was evaluated for the presence or absence of the following signs [4, 21]: $\mathrm{RV}>30 \mathrm{~mm}$ or $\mathrm{RV} / \mathrm{LV}$ end-diastolic ratio $>1$ from the apical 4-CH view; dyskinesia or hypokinesia of the free right ventricular wall; hypokinesia of the infundibular RV region with normal contraction of the RV apex (McConnell sign); tricuspid annular plane systolic excursion $<15 \mathrm{~mm}$; RV/atrial gradient $>30 \mathrm{mmHg}$. A diagnosis of $\mathrm{RV}$ dysfunction was established in the presence of two or more of these criteria [21,
22]. In addition, if bulging of the interventricular septum into the LV was observed, RVD was classified as severe [22, 23]. All other forms of RVD were classified as moderate.

\section{CT protocol}

All standard pulmonary CT angiography examinations were performed on multi-detector CT (MDCT) systems. 40 patients were examined using a 16-slice MDCT system (Somatom Emotion; Siemens Healthcare, Forchheim, Germany). The remaining 37 patients were examined using a 64-slice dualsource CT system (Somatom Definition; Siemens Healthcare). $100 \mathrm{~mL}$ iodinated contrast material (Imeron 400; Bracco Imaging S.p.A., Milan, Italy) was injected in an antecubital vein using a power injector (Stellant D; Medrad, Warrendale, PA, USA) at a rate of $4 \mathrm{~mL} \cdot \mathrm{s}^{-1}$, followed by a $20-\mathrm{mL}$ saline chaser. In all examinations the entire chest was scanned in a caudo-cranial direction during an inspiratory breath hold.

\section{CT analysis}

All CT studies were analysed on a multi-modality 3D-enabled workstation (Syngo VE36A; Siemens Healthcare). CT studies were evaluated by two radiologists (C. Fink and T. Henzler) in consensus, who were blinded to the echocardiographic and laboratory results.

\section{RVILV axial diameter ratio}

The axial section displaying the maximal distance between the ventricular endocardium and the interventricular septum, perpendicular to the long axis of the heart, was identified for both the right and left ventricle. The maximum short axis diameters were measured for the right ventricle and the left ventricle, and the $\mathrm{RV} / \mathrm{LV}$ axial ratio was subsequently calculated (fig. 1a).

\section{$\mathrm{RV} / \mathrm{LV}$ 4-CH view ratio}

4- $\mathrm{CH}$ views were reconstructed as previously described [12]. The two levels on the reconstructed $4-\mathrm{CH}$ views that showed the maximal distance between the ventricular endocardium and the interventricular septum for the right ventricle and left ventricle were identified. The RV and $\mathrm{LV} 4-\mathrm{CH}$ diameters were measured, and from this the $\mathrm{RV} / \mathrm{LV} 4-\mathrm{CH}$ ratio was calculated (fig. 1b).
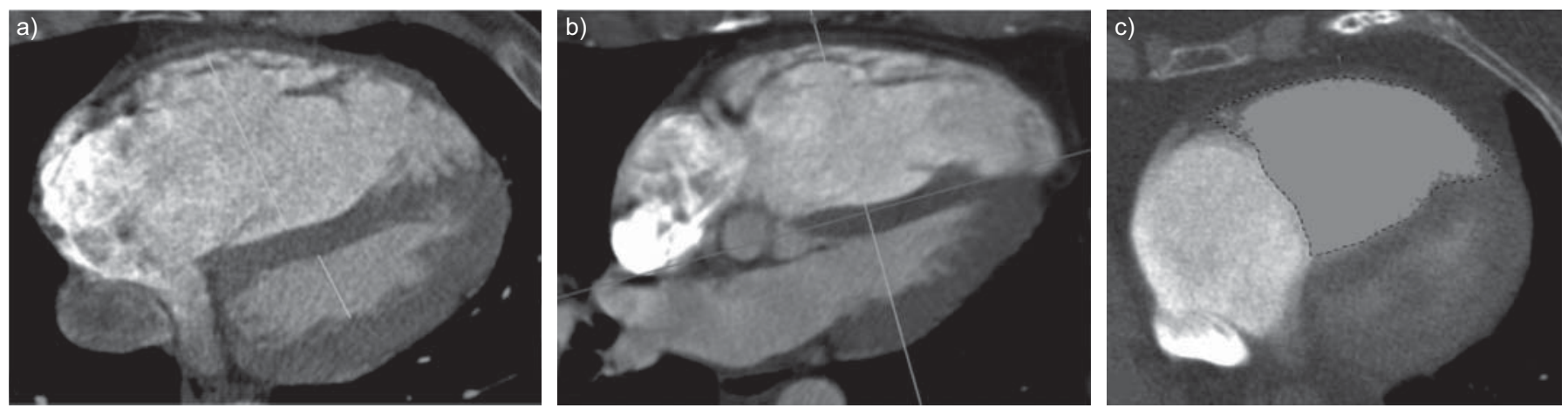

FIGURE 1. Pulmonary computed tomography angiography study in a 67-yr-old female with acute pulmonary embolism. Right ventricular/left ventricular ratio assessed on a) an axial section and b) on a reconstructed four-chamber view. The widest diameter of each ventricle was not necessarily located on the same section. c) Volumetric analysis of the right ventricle using a threshold-dependent algorithm. The manually segmented contours were automatically propagated to the neighbouring sections. Voxels with attenuation in the range of myocardium were automatically excluded from the volume analysis, after the attenuation of the septal myocardium was measured three times for each patient. 


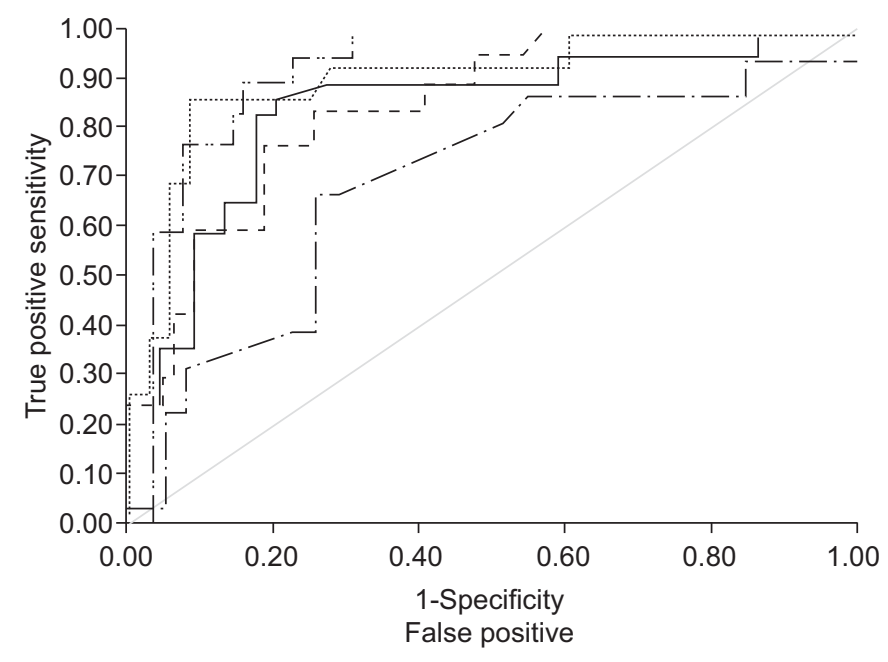

FIGURE 2. Receiver operating characteristic curves of the three different computed tomography parameters and cardiac biomarkers for predicting right ventricular dysfunction on echocardiography in patients with acute pulmonary embolism. —: right ventricular (RV)/left ventricular (LV)axial, AUC $0.84 ; \cdots \cdot$ : RV/LV4chamber, AUC 0.87; -..-..-: RV/LVvolume, AUC 0.93; - - - -: N-terminal pro-brain natriuretic peptide, AUC $0.83 ;-\cdot-\cdot-$ : troponin I, AUC 0.70

\section{$R V / L V$ volume ratio}

3D-volumetric analysis of both ventricles was performed by using dedicated volume analysis software (Syngo VE31A, Siemens Healthcare). The endocardial contours were semiautomatically segmented from the valvular plane to the apex of both ventricles and the RV/LVvolume ratio was subsequently calculated as previously described (fig. 1c) [15].

\section{Laboratory measurements}

NT-pro-BNP and troponin I serum levels were quantified from a venous blood sample, which was drawn within $24 \mathrm{~h}$ after the diagnosis of pulmonary embolism. Plasma NT-pro-BNP concentration was determined using an NT-pro-BNP enzyme immunoassay and a Dimension RxL analyser (Siemens Healthcare Diagnostics, Eschborn, Germany). The manufacturer's proposed decision threshold for excluding heart failure in patients without renal insufficiency is $84 \mathrm{ng} \cdot \mathrm{L}^{-1}$ in males and $155 \mathrm{ng} \cdot \mathrm{L}^{-1}$ in females aged $\leqslant 50 \mathrm{yrs}$, and $194 \mathrm{ng} \cdot \mathrm{L}^{-1}$ in males and $222 \mathrm{ng} \cdot \mathrm{L}^{-1}$ in females $>50$ yrs.

Troponin I levels were measured with a two-site immunoenzymatic immunoassay (Access AccuTnI; Beckmann Coulter
GmbH Diagnostic, Krefeld, Germany). According to the manufacturer's instructions, the cut-off point for an upper limit of normal (with an assay coefficient of variation $<10 \%$ ) was $0.06 \mathrm{~g} \cdot \mathrm{L}^{-1}$.

\section{Statistical analysis}

Statistical analysis was performed using JMP 7.0 (SAS Institute, Cary, NC, USA). Continuous variables are expressed as mean $\pm \mathrm{SD}$. The Shapiro-Wilk test was applied to determine probability distribution; a two-tailed paired t-test was subsequently used to compare groups with normal distribution, while the Mann-Whitney U-test was used if the data were not normally distributed. The Chi-squared test was applied for dichotomous variables. Pearson's correlation was used to correlate serum levels of NT-pro-BNP with RV/LVaxial, RV/ LV4-CH, RV/LVvolume and echocardiographic assessment of RVD. To determine the diagnostic accuracy of cardiac biomarkers and CT parameters for RVD, receiver operating characteristic (ROC) curves were analysed and areas under the curve (AUCs) were calculated. Differences between AUC values were compared using the Hanley and McNeil method. Multivariate analysis was performed with logistic regression analysis using block entry of the following variables: NT-pro$\mathrm{BNP}$, troponin I, RV/LVaxial, RV/LV4-CH and RV/LVvolume. The results are presented as estimated odds ratios (ORs) and relative risk with the corresponding $95 \%$ confidence intervals. A two-tailed $\mathrm{p}$-value of $<0.05$ was considered statistically significant.

\section{RESULTS}

Among the 77 patients with acute pulmonary embolism, congestive heart failure was present in 13 patients, cancer in 11, myocardial infarction in eight, chronic kidney disease in four, pulmonary hypertension in four and sepsis in two patients. Echocardiography showed RVD in 27 (35\%) out of 77 patients, of whom $15(56 \%)$ were classified as severe and 12 (44\%) as moderate.

\section{Overall detection of RVD}

Patients with RVD showed significantly higher RV/LV ratios and cardiac biomarker levels compared to those without RVD (RV/LVaxial $1.68 \pm 0.84$ versus $1.00 \pm 0.21(\mathrm{p}=0.003) ; \mathrm{RV} / \mathrm{LV} 4-\mathrm{CH}$ $1.52 \pm 0.45$ versus $1.01 \pm 0.21(\mathrm{p}=0.002) ; \mathrm{RV} / \mathrm{LV}$ volume $1.97 \pm 0.53$ versus $1.07 \pm 0.52(\mathrm{p}=0.0001)$; serum NT-pro-BNP 6,372 $\pm 2,319$ versus $1,032 \pm 1,559 \mathrm{ng} \cdot \mathrm{L}^{-1}(\mathrm{p}=0.002)$; troponin I $0.179 \pm 0.411$ versus $\left.0.061 \pm 0.176 \mathrm{~g} \cdot \mathrm{L}^{-1}(\mathrm{p}=0.0375)\right)$.

TABLE 1 Diagnostic accuracy of right ventricular (RV)/left ventricular (LV)axial, RV/LV4-CH, RV/LVvolume and cardiac biomarkers for the detection of right ventricular dysfunction

\begin{tabular}{|c|c|c|c|c|c|c|}
\hline RV/LVaxial & 82 & 83 & 68 & 92 & 0.84 & 1.18 \\
\hline RV/LVvolume & 88 & 85 & 82 & 95 & 0.93 & 1.34 \\
\hline NT-pro-BNP ng. $\mathrm{L}^{-1}$ & 75 & 80 & 60 & 88 & 0.83 & 1617 \\
\hline Troponin I $\mathbf{g} \cdot \mathrm{L}^{-1}$ & 67 & 72 & 56 & 80 & 0.70 & 0.07 \\
\hline
\end{tabular}

4-CH: four-chamber view; PPV: positive predictive value; NPV: negative predictive value; AUC: area under the curve; NT-pro-BNP: N-terminal pro-brain natriuretic peptide. 


\begin{tabular}{|c|c|c|c|c|c|c|}
\hline \multirow[t]{2}{*}{ TABLE 2} & \multicolumn{6}{|c|}{$\begin{array}{l}\text { Diagnostic accuracy of right ventricular (RV)/left ventricular (LV)axial, RV/LV4-CH and RV/LVvolume in combination with } \\
\text { cardiac biomarkers for the detection of right ventricular dysfunction }\end{array}$} \\
\hline & Sensitivity \% & Specificity \% & PPV \% & NPV \% & AUC & Cut-off value \\
\hline \multicolumn{7}{|c|}{ NT-pro-BNP ng. $\mathrm{L}^{-1}$ with } \\
\hline RV/LVaxial & 79 & 87 & 69 & 92 & 0.87 & $1.18 / 1617$ \\
\hline $\mathrm{RV} / \mathrm{LV} 4-\mathrm{CH}$ & 84 & 92 & 77 & 93 & 0.90 & $1.29 / 1617$ \\
\hline \multicolumn{7}{|c|}{ Troponin I $\mathrm{g} \cdot \mathrm{L}^{-1}$ with } \\
\hline RV/LVaxial & 85 & 78 & 67 & 91 & 0.85 & $1.18 / 0.07$ \\
\hline $\mathrm{RV} / \mathrm{LV}_{4}-\mathrm{CH}$ & 89 & 80 & 71 & 93 & 0.88 & $1.29 / 0.07$ \\
\hline RV/LVvolume & 93 & 90 & 84 & 96 & 0.93 & $1.34 / 0.07$ \\
\hline
\end{tabular}

4-CH: four-chamber view; PPV: positive predictive value; NPV: negative predictive value; AUC: area under the curve; NT-pro-BNP: N-terminal pro-brain natriuretic peptide.

The correlation coefficient of the three different CT parameters with serum NT-pro-BNP was weak for RV/LVaxial with NTpro-BNP $(\mathrm{r}=0.38)$, moderate for $\mathrm{RV} / \mathrm{LV} 4-\mathrm{CH}$ with NT-pro-BNP $(\mathrm{r}=0.52)$, and good for $\mathrm{RV} / \mathrm{LV}$ volume with NT-pro-BNP $(r=0.68)$. No correlation was found between the three different $\mathrm{CT}$ parameters with troponin I: $\mathrm{RV} / \mathrm{LV}$ axial with troponin I $(\mathrm{r}=0.10)$, RV/LV4-CH with troponin I $(r=0.12)$, and $\mathrm{RV} /$ LVvolume with troponin I $(r=0.19)$.

The AUCs of RV/LVaxial, RV/LV4-CH, RV/LVvolume, serum NT-pro-BNP and troponin I for predicting RVD were 0.84, $0.87,0.93,0.83$ and 0.70 , respectively (fig. 2). ROC analysis of CT parameters and cardiac biomarkers revealed the following cut-off values for the prediction of RVD: 1.18 for RV/LVaxial, 1.29 for $\mathrm{RV} / \mathrm{LV} 4-\mathrm{CH}, 1.34$ for $\mathrm{RV} / \mathrm{LV}_{\text {volume, }} 1617 \mathrm{ng} \cdot \mathrm{L}^{-1}$ for NT-pro-BNP and $0.07 \mathrm{~g} \cdot \mathrm{L}^{-1}$ for troponin I (fig. 2). Table 1 summarises the diagnostic characteristics of RV/LVaxial, RV/ LV4-CH, RV/LVvolume, serum NT-pro-BNP and troponin I using the specified cut-off values.

A combination of NT-pro-BNP and the three different CT parameters increased the AUC of RV/LVaxial, RV/LV4-CH and $\mathrm{RV} / \mathrm{LV}$ volume to $0.87,0.90$ and 0.95 , respectively (all $\mathrm{p}>0.05$ ). Table 2 summarises the diagnostic accuracy of RV/LVaxial, $\mathrm{RV} / \mathrm{LV} 4-\mathrm{CH}$ and RV/LVvolume in combination with NT-proBNP for the detection of RVD. A combination of troponin I and the three different CT parameters increased the AUC of RV/ LVaxial, RV/LV4-CH and RV/LVvolume to 0.85, 0.88 and 0.93, respectively (all $\mathrm{p}>0.05$ ). Table 2 summarises the diagnostic accuracy of $\mathrm{RV} / \mathrm{LV}$ axial, $\mathrm{RV} / \mathrm{LV} 4-\mathrm{CH}$ and $\mathrm{RV} / \mathrm{LV}$ volume in combination with troponin I serum levels for the detection of moderate RVD.

Multiple logistic regression analysis revealed that RV/LVaxial (OR 37.5, 95\% CI 8-190; $\mathrm{p}=0.0001)$, RV/LV4-CH (OR 45.7, 95\% CI 10-215; $\mathrm{p}=0.0001$ ), RV /LVvolume (OR 67.5, 95\% CI 12-370; $\mathrm{p}=0.0001)$, NT-pro-BNP (OR 12, 95\% CI 3.0-47.2; $\mathrm{p}=0.002)$ and troponin I (OR 5, 95\% CI 1.6-15.9; $\mathrm{p}=0.019)$ were all independent predictors of RVD.

\section{Detection of moderate RVD}

ROC analysis of the patient group with moderate RVD revealed the following cut-off values for RV/LVaxial, RV/ LV4-CH, RV/LVvolume, serum NT-pro-BNP and troponin I for detecting patients with echocardiographically confirmed moderate RVD: $1.23,1.31,1.33,1,427 \mathrm{ng} \cdot \mathrm{L}^{-1}$ and $0.09 \mathrm{~g} \cdot \mathrm{L}^{-1}$, respectively. Using these cut-off values, the AUCs of RV/ LVaxial, RV/LV4-CH, RV/LVvolume, serum NT-pro-BNP and troponin I were $0.87,0.89,0.90,0.80$ and 0.71 , respectively. Table 3 summarises the diagnostic accuracy of RV/LVaxial, $\mathrm{RV} / \mathrm{LV} 4-\mathrm{CH}, \mathrm{RV} / \mathrm{LV}$ volume, serum NT-pro-BNP and troponin I for the detection of moderate RVD.

A combination of NT-pro-BNP and the three different CT parameters increased the AUC of RV/LVaxial, RV/LV4-CH and $\mathrm{RV} / \mathrm{LV}$ volume to $0.90,0.91$ and 0.93 , respectively (all $\mathrm{p}>0.05$ ). Table 4 summarises the diagnostic accuracy of RV/LVaxial, RV/ LV4-CH and RV/LVvolume in combination with NT-pro-BNP

\begin{tabular}{|c|c|c|c|c|c|c|}
\hline & Sensitivity \% & Specificity \% & PPV \% & NPV \% & AUC & Cut-off value \\
\hline RV/LVaxial & 83 & 80 & 50 & 95 & 0.87 & 1.23 \\
\hline RV/LV4-CH & 83 & 88 & 63 & 96 & 0.89 & 1.31 \\
\hline RV/LVvolume & 92 & 88 & 65 & 98 & 0.90 & 1.33 \\
\hline
\end{tabular}




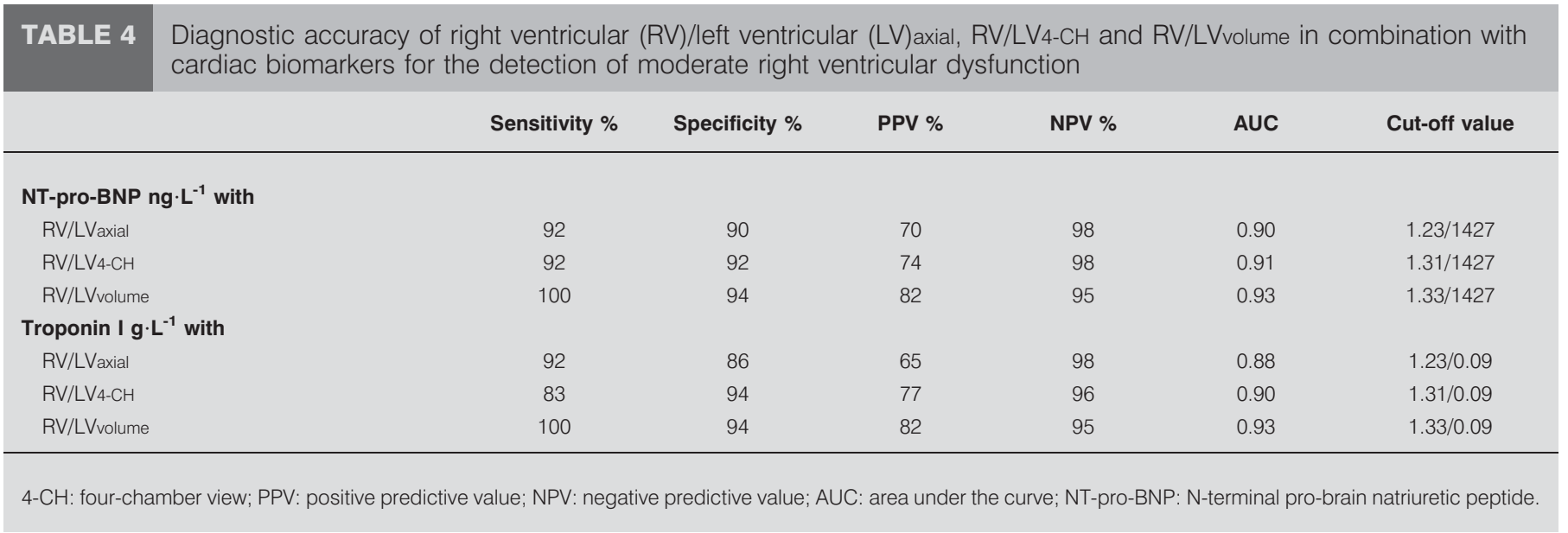

serum levels for the detection of moderate RVD. A combination of troponin I and the three different CT parameters increased the AUC of RV/LVaxial, RV/LV4-CH and RV/LVvolume to 0.88, 0.90 and 0.91 , respectively (all $\mathrm{p}>0.05$ ). Table 4 summarises the diagnostic accuracy of $\mathrm{RV} / \mathrm{LV}$ axial, $\mathrm{RV} / \mathrm{LV} 4-\mathrm{CH}$ and $\mathrm{RV} /$ $\mathrm{LV}_{\text {volume }}$ in combination with troponin I serum levels for the detection of moderate RVD.

\section{Detection of severe RVD}

ROC analysis of the patient group with severe RVD revealed the following cut-off values for RV/LVaxial, RV/LV4-CH, RV/ LVvolume, serum NT-pro-BNP and troponin for detecting patients with echocardiographically confirmed severe RVD: $1.28,1.39,1.72,1,840 \mathrm{ng} \cdot \mathrm{L}^{-1}$ and $0.1 \mathrm{~g} \cdot \mathrm{L}^{-1}$, respectively. Using these cut-off values, the AUCs of RV/LVaxial, RV/LV4-CH, RV/ LVvolume, serum NT-pro-BNP and troponin I were $0.80,0.79$, $0.94,0.93$ and 0.73 , respectively (fig. 3). Table 5 summarises the diagnostic accuracy of RV/LVaxial, RV/LV4-CH, RV/LVvolume,

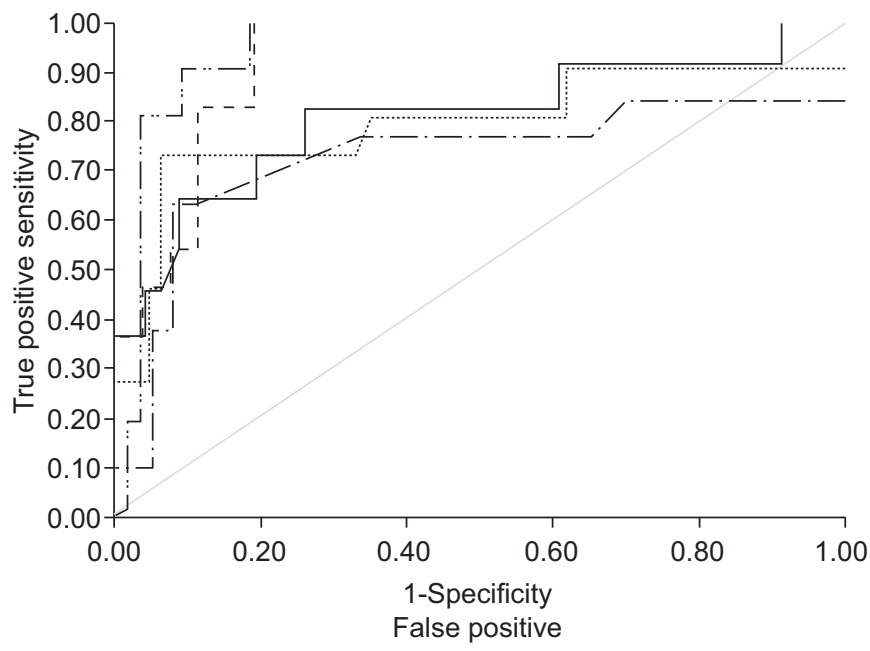

FIGURE 3. Receiver operating characteristic curves of the three different computed tomography parameters and cardiac biomarkers for the prediction of echocardiographically confirmed severe right ventricular dysfunction in patients with acute pulmonary embolism. — : right ventricular (RV)/left ventricular (LV)axial, AUC $0.80 ; \cdots \cdot$ : RV/LV4-chamber, AUC 0.79; -.....-: RV/LVvolume, AUC 0.94; - - - -: Nterminal pro-brain natriuretic peptide, AUC 0.93; --_--: troponin I, AUC 0.73 serum NT-pro-BNP and troponin I for the detection of severe RVD.

A combination of NT-pro-BNP and the three different CT parameters increased the AUC of RV/LVaxial, RV/LV4-CH and $\mathrm{RV} / \mathrm{LV}$ volume statistically significantly to $0.91,0.93$, and 0.98 , respectively (all $\mathrm{p}<0.05$ ). Table 6 summarises the diagnostic accuracy of $\mathrm{RV} / \mathrm{LV}$ axial, $\mathrm{RV} / \mathrm{LV} 4-\mathrm{CH}$ and $\mathrm{RV} / \mathrm{LV}_{\text {volume }}$ in combination with NT-pro-BNP serum levels for the detection of severe RVD. A combination of troponin I and the three different CT parameters increased the AUC of RV/LVaxial,

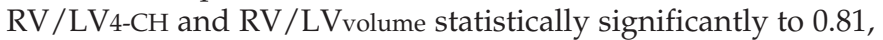
0.80 and 0.94 , respectively (all $p<0.05$ ). Table 6 summarises the diagnostic accuracy of $\mathrm{RV} / \mathrm{LV}$ axial, $\mathrm{RV} / \mathrm{LV} 4-\mathrm{CH}$ and $\mathrm{RV} /$

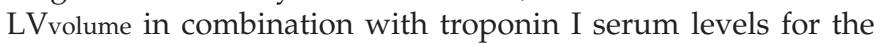
detection of severe RVD.

\section{DISCUSSION}

We have shown that, in a consecutive cohort of unselected patients with acute pulmonary embolism, 3D measurements of ventricular volumes and elevated cardiac biomarker serum levels are superior to uni-dimensional $\mathrm{RV} / \mathrm{LV}$ diameter ratios for the prediction of RVD. Moreover, we have shown that a combination of RV/LVvolume and cardiac biomarker measurements increased the diagnostic accuracy when compared to either parameter alone.

Echocardiography is considered the reference standard for the assessment of RVD in patients with pulmonary embolism because it can assess RV size and function, as well as measuring pulmonary artery pressures. Echocardiography can be performed at the bedside and allows for repetitive noninvasive assessment of haemodynamic status and response to treatment. However, accurate echocardiographic imaging of the RV free wall can be technically challenging and, at times, impossible in a patient with dyspnoea, especially in the presence of obesity or chronic lung disease [24]. In addition, at many institutions the availability of this test is limited to week day daytime hours, whereas pulmonary CT angiography typically has much greater circadian availability, even in small centres [24]. The measurement of serum NT-pro-BNP levels has become routinely available at most clinical laboratories. Accordingly, the combination of cardiac biomarkers and quantitative cardiac CT parameters from pulmonary $\mathrm{CT}$ 


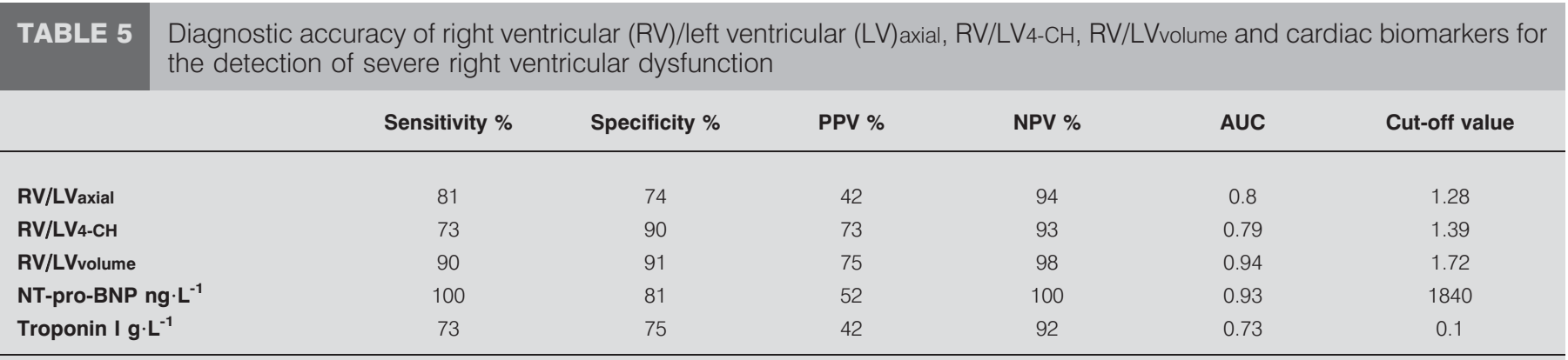

4-CH: four-chamber view; PPV: positive predictive value; NPV: negative predictive value; AUC: area under the curve; NT-pro-BNP: N-terminal pro-brain natriuretic peptide.

angiography could be a cost-effective alternative for detecting RVD and for stratifying patient risk in clinical scenarios where echocardiography is not readily available.

An increased $\mathrm{RV} / \mathrm{LV}$ diameter ratio on pulmonary CT angiography has been suggested in several studies [7-9, 12, 13,25 ] as a surrogate marker for RVD and shown to be a predictor of short-term mortality and adverse clinical events in patients with acute pulmonary embolism. However, only three studies with a limited number of patients have directly compared CT findings with echocardiography [10, 17, 18]. LIM et al. [18] retrospectively reviewed CT studies of 14 patients with acute, massive pulmonary embolism during a 52month period, with CT showing a sensitivity of $91.6 \%$ and a specificity of $100 \%$ for the detection of RVD compared to echocardiography. Likewise, CONTRACTOR et al. [17] evaluated 25 patients with pulmonary embolism and showed a sensitivity of $78 \%$ and a specificity of $100 \%$. However, those studies limited their evaluation to qualitative parameters (right ventricular dilation or septal bowing) for diagnosing RVD. Quantification of RV/LV ratios, as performed by MANSENCAL et al. [10], may provide a more reproducible parameter for identifying RVD. Their study evaluated 46 consecutive patients with pulmonary embolism who underwent pulmonary CT angiography and echocardiography. An $\mathrm{RV} / \mathrm{LV}$ area ratio $>1$ on CT was shown to provide $88 \%$ sensitivity and $88 \%$ specificity for diagnosing RVD compared with echocardiography, which is comparable to the performance of $\mathrm{RV} / \mathrm{LV}$ volume found in our study.
In our study, $\mathrm{RV} / \mathrm{LV}$ volume was more accurate than both unidimensional RV/LV diameter ratios (RV/LVaxial and RV/LV4$\mathrm{CH})$ for predicting echocardiographically confirmed RVD in patients with acute pulmonary embolism. These findings support those of previous feasibility studies in which RV/

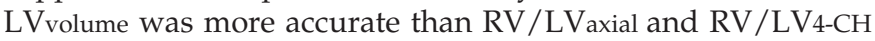
for differentiating patients with and without central pulmonary embolism $[14,15]$. The better correlation of $\mathrm{RV} / \mathrm{LV}$ volume with NT-pro-BNP serum levels compared to RV/LVaxial and $\mathrm{RV} / \mathrm{LV} 4-\mathrm{CH}$ may be explained by the notion that volumetric analysis of the entire ventricle may better reflect right ventricular overload and, thus, may be superior for assessing the myocardial strain which causes NT-pro-BNP and troponin I release. Although $\mathrm{RV} / \mathrm{LV} 4-\mathrm{CH}$ and $\mathrm{RV} / \mathrm{LV}$ volume showed higher AUC values than NT-pro-BNP for the detection of all patients with RVD, NT-pro-BNP showed the highest sensitivity when compared to all CT parameters for detecting those patients with severe RVD. Troponin I showed a lower diagnostic performance when compared to RV/LVvolume and NT-pro-BNP whereas a combination between RV/LVvolume and troponin I led to almost similar diagnostic results to those of a combination of $\mathrm{RV} / \mathrm{LV}_{\text {volume }}$ and NT-pro-BNP. Since troponin I is a marker of myocardial cell damage, significant serum elevation might not be found within the first $24 \mathrm{~h}$ after pulmonary embolism in patients with only moderate RVD. However, newer assays for cardiac troponin that have been developed recently are able to detect changes in concentration of the biomarker at or below the 99th percentile for a normal population [26]. Therefore, future studies have to investigate

\begin{tabular}{|c|c|c|c|c|c|c|}
\hline \multirow[t]{2}{*}{ TABLE 6} & \multicolumn{6}{|c|}{$\begin{array}{l}\text { Diagnostic accuracy of right ventricular (RV)/left ventricular (LV)axial, RV/LV4-CH and RV/LVvolume in combination with } \\
\text { cardiac biomarkers for the detection of severe right ventricular dysfunction }\end{array}$} \\
\hline & Sensitivity \% & Specificity \% & PPV \% & NPV \% & AUC & Cut-off value \\
\hline \multicolumn{7}{|c|}{ NT-pro-BNP $n g \cdot L^{-1}$ with } \\
\hline RV/LVaxial & 100 & 98 & 90 & 100 & 0.91 & $1.28 / 1840$ \\
\hline $\mathrm{RV} / \mathrm{LV} 4-\mathrm{CH}$ & 100 & 98 & 90 & 100 & 0.93 & $1.39 / 1840$ \\
\hline RV/LVaxial & 87 & 89 & 67 & 97 & 0.81 & $1.28 / 0.1$ \\
\hline $\mathrm{RV} / \mathrm{LV} 4-\mathrm{CH}$ & 87 & 87 & 65 & 97 & 0.80 & $1.39 / 0.1$ \\
\hline RV/LVvolume & 100 & 98 & 94 & 100 & 0.94 & $1.72 / 0.1$ \\
\hline
\end{tabular}


whether new high-sensitivity troponin assays are superior to conventional troponin I for the diagnosis of RVD in patients with pulmonary embolism.

Serum NT-pro-BNP and troponin I levels have been proposed as a non-imaging biomarker for improved risk stratification in patients with acute pulmonary embolism. A recent metaanalysis on the prognostic value of NT-pro-BNP for predicting 30-day adverse events showed an overall sensitivity and specificity of $93 \%$ and $58 \%$, respectively. The negative and positive predictive values were $81 \%$ and $63 \%$, respectively [1] These results document the high sensitivity and the favourable negative predictive value of NT-pro-BNP assessment. In another meta-analysis about the prognostic value of troponins in acute pulmonary embolism, BECATTINI et al. [27] found an unadjusted OR of 5.2 (95\% CI 3.3-8.4) of elevated cardiac troponin for the prediction of death in patients with pulmonary embolism. Similar values were observed in our study for the prediction of severe RVD (table 2). BINDER et al. [28] also demonstrated that NT-pro-BNP combined with echocardiography may reliably identify both low-risk and high-risk patients with pulmonary embolism. These data suggest that while cardiac biomarkers do not have high enough specificity as a stand-alone test to identify high-risk patients, they may have value in combination with other diagnostic tests, such as imaging. Therefore, we evaluated three reported markers of poor prognosis (RVD as seen on pulmonary CT angiography, and serum NT-pro-BNP and troponin I) and compared them with the established first-line risk stratification tool, echocardiography. VUILLEUMIER et al. [29] evaluated the correlation between NT-pro-BNP and RV/ LV4-CH and found a correlation of 0.36 between both parameters, which is similar to our uni-dimensional measurement results. However, their study did not evaluate the combination of both parameters and RVD was not confirmed by echocardiography.

There are several limitations to our study that have to be considered. First, we did not exclude patients with other underlying disease states that may have led to an increase in serum NT-pro-BNP and troponin I levels. Moreover, we based our observations on routine pulmonary CT angiography techniques rather than ECG-synchronised scan protocols. Non ECG-synchronised CT has some potential limitations for measuring ventricular chamber size, because the images are not acquired during a specific phases of the cardiac cycle. However, it has been previously demonstrated that the use of ECG-synchronised CT protocols is only of limited incremental diagnostic value when compared to routine techniques [30]. More importantly, because of the additional radiation exposure involved with retrospective ECG-gated techniques of the whole chest, this approach is not currently used for routine pulmonary embolism imaging [31], whereas our results obtained in non ECG-gated pulmonary CT angiography studies are directly transferable to clinical practice. However, recently published studies have demonstrated that high-pitch pulmonary CT angiography, as well as prospectively ECGgated pulmonary CT angiography protocols, are able to acquire studies with fewer motion artefacts and an even lower radiation dose when compared to standard non ECG-gated pulmonary CT angiography protocols [32, 33].
A second limitation of our findings concerns the broad clinical applicability. Although we observed superiority of RV/

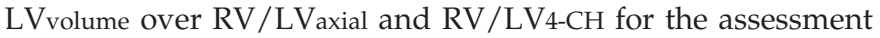
of RVD, it has to be mentioned that simple diameter measurements are less time consuming when compared to a volumetric analysis that requires dedicated software tools. Thus, it remains unclear whether the technique is suitable for smaller medical centres, which may not have the software or personnel recourses.

Finally, we did not evaluate adverse outcomes of our patients as this study aimed to compare the diagnostic accuracy of cardiac CT parameters and cardiac biomarkers for the detection of RVD, with echocardiography as the established imaging modality to assess RVD in patients with acute pulmonary embolism. Future studies should evaluate whether a combination of cardiac CT parameters, in particular RV/ LVvolume, and cardiac biomarkers allow an improved prediction of adverse outcomes in patients with acute pulmonary embolism compared with echocardiography.

In conclusion, CT-derived RV/LVvolume compares favourably with echocardiography for the diagnosis of RVD in patients with acute pulmonary embolism and shows good correlation with cardiac biomarker serum levels. A combination of RV/ LV volume and NT-pro-BNP or troponin I has higher diagnostic accuracy than either parameter in isolation. Accordingly, quantitative cardiac CT parameters obtained from pulmonary CT anigography in combination with cardiac biomarker measurements could be used as an alternative to echocardiography for the detection of right ventricular dysfunction in patients with acute pulmonary embolism.

\section{STATEMENT OF INTEREST}

A statement of interest for U.J. Schoepf can be found at www.erj. ersjournals.com/site/misc/statements.xhtml

\section{REFERENCES}

1 Sanchez O, Trinquart L, Colombet I, et al. Prognostic value of right ventricular dysfunction in patients with haemodynamically stable pulmonary embolism: a systematic review. Eur Heart J 2008; 29: 1569-1577.

2 Kreit JW. The impact of right ventricular dysfunction on the prognosis and therapy of normotensive patients with pulmonary embolism. Chest 2004; 125: 1539-1545.

3 Leacche M, Unic D, Goldhaber SZ, et al. Modern surgical treatment of massive pulmonary embolism: results in 47 consecutive patients after rapid diagnosis and aggressive surgical approach. J Thorac Cardiovasc Surg 2005; 129: 1018-1023.

4 Grifoni S, Olivotto I, Cecchini P, et al. Short-term clinical outcome of patients with acute pulmonary embolism, normal blood pressure, and echocardiographic right ventricular dysfunction. Circulation 2000; 101: 2817-2822.

5 Kasper W, Konstantinides S, Geibel A, et al. Prognostic significance of right ventricular afterload stress detected by echocardiography in patients with clinically suspected pulmonary embolism. Heart 1997; 77: 346-349.

6 Ribeiro A, Lindmarker $\mathrm{P}$, Juhlin-Dannfelt A, et al. Echocardiography Doppler in pulmonary embolism: right ventricular dysfunction as a predictor of mortality rate. Am Heart J 1997; 134: 479-487.

7 Araoz PA, Gotway MB, Harrington JR, et al. Pulmonary embolism: prognostic CT findings. Radiology 2007; 242: 889-897. 
8 Ghaye B, Ghuysen A, Willems V, et al. Severe pulmonary embolism:pulmonary artery clot load scores and cardiovascular parameters as predictors of mortality. Radiology 2006; 239: 884-891.

9 Ghuysen A, Ghaye B, Willems V, et al. Computed tomographic pulmonary angiography and prognostic significance in patients with acute pulmonary embolism. Thorax 2005; 60: 956-961.

10 Mansencal N, Joseph T, Vieillard-Baron A, et al. Diagnosis of right ventricular dysfunction in acute pulmonary embolism using helical computed tomography. Am J Cardiol 2005; 95: 1260-1263.

11 Quiroz R, Kucher N, Schoepf UJ, et al. Right ventricular enlargement on chest computed tomography: prognostic role in acute pulmonary embolism. Circulation 2004; 109: 2401-2404.

12 Schoepf UJ, Kucher N, Kipfmueller F, et al. Right ventricular enlargement on chest computed tomography: a predictor of early death in acute pulmonary embolism. Circulation 2004; 110: 3276-3280.

13 van der Meer RW, Pattynama PM, van Strijen MJ, et al. Right ventricular dysfunction and pulmonary obstruction index at helical CT: prediction of clinical outcome during 3-month followup in patients with acute pulmonary embolism. Radiology 2005; 235: 798-803.

14 Dogan H, Kroft LJ, Huisman MV, et al. Right ventricular function in patients with acute pulmonary embolism: analysis with electrocardiography-synchronized multi-detector row CT. Radiology 2007; 242: 78-84.

15 Henzler T, Krissak R, Reichert M, et al. Volumetric analysis of pulmonary CTA for the assessment of right ventricular dysfunction in patients with acute pulmonary embolism. Acad Radiol 2010; 17: 309-315.

16 van der Bijl N, Klok FA, Huisman MV, et al. Measurement of right and left ventricular function by ECG-synchronized CT in patients with acute pulmonary embolism: usefulness for predicting shortterm outcome. Chest 2011; 140: 1008-1015.

17 Contractor S, Maldjian PD, Sharma VK, et al. Role of helical CT in detecting right ventricular dysfunction secondary to acute pulmonary embolism. J Comput Assist Tomogr 2002; 26: 587-591.

18 Lim KE, Chan CY, Chu PH, et al. Right ventricular dysfunction secondary to acute massive pulmonary embolism detected by helical computed tomography pulmonary angiography. Clin Imaging 2005; 29: 16-21.

19 Klok FA, Mos IC, Huisman MV. Brain-type natriuretic peptide levels in the prediction of adverse outcome in patients with pulmonary embolism: a systematic review and meta-analysis. Am J Respir Crit Care Med 2008; 178: 425-430.

20 Horlander KT, Leeper KV. Troponin levels as a guide to treatment of pulmonary embolism. Curr Opin Pulm Med 2003; 9: 374-377.

21 Lang RM, Bierig M, Devereux RB, et al. Recommendations for chamber quantification: a report from the American Society of Echocardiography's Guidelines and Standards Committee and the
Chamber Quantification Writing Group, developed in conjunction with the European Association of Echocardiography, a branch of the European Society of Cardiology. J Am Soc Echocardiogr 2005; 18: 1440-1463.

22 Vieillard-Baron A, Prin S, Chergui K, et al. Echo-Doppler demonstration of acute cor pulmonale at the bedside in the medical intensive care unit. Am J Respir Crit Care Med 2002; 166: 1310-1319.

23 Logeart D, Lecuyer L, Thabut G, et al. Biomarker-based strategy for screening right ventricular dysfunction in patients with nonmassive pulmonary embolism. Intensive Care Med 2007; 33: 286-292.

24 Goldhaber SZ. Advanced treatment strategies for acute pulmonary embolism, including thrombolysis and embolectomy. J Thromb Haemost 2009; 7: Suppl. 1, 322-327.

25 Araoz PA, Gotway MB, Trowbridge RL, et al. Helical CT pulmonary angiography predictors of in-hospital morbidity and mortality in patients with acute pulmonary embolism. J Thorac Imaging 2003; 18: 207-216.

26 Reichlin T, Hochholzer W, Bassetti S, et al. Early diagnosis of myocardial infarction with sensitive cardiac troponin assays. N Engl J Med 2009; 361: 858-867.

27 Becattini C, Vedovati MC, Agnelli G. Prognostic value of troponins in acute pulmonary embolism: a meta-analysis. Circulation 2007; 116: 427-433.

28 Binder L, Pieske B, Olschewski M, et al. N-terminal pro-brain natriuretic peptide or troponin testing followed by echocardiography for risk stratification of acute pulmonary embolism. Circulation 2005; 112: 1573-1579.

29 Vuilleumier N, Righini M, Perrier A, et al. Correlation between cardiac biomarkers and right ventricular enlargement on chest CT in non massive pulmonary embolism. Thromb Res 2008; 121: 617624.

$30 \mathrm{Lu} \mathrm{MT}, \mathrm{Cai} \mathrm{T}$, Ersoy $\mathrm{H}$, et al. Interval increase in right-left ventricular diameter ratios at $\mathrm{CT}$ as a predictor of 30-day mortality after acute pulmonary embolism: initial experience. Radiology 2008; 246: 281-287.

31 Schoepf UJ, Costello P. CT angiography for diagnosis of pulmonary embolism: state of the art. Radiology 2004; 230: 329-337.

32 Shuman WP, Leipsic JA, Busey JM, et al. Prospectively ECG gated CT pulmonary angiography versus helical ungated CT pulmonary angiography: impact on cardiac related motion artefacts and patient radiation dose. Eur J Radiol 2011 [Epub ahead of print DOI: 10.1016/j.ejrad.2011.06.017].

33 Karakas M, Januzzi JL Jr, Meyer J, et al. Copeptin does not add diagnostic information to high-sensitivity troponin $\mathrm{T}$ in low- to intermediate-risk patients with acute chest pain: results from the rule out myocardial infarction by computed tomography (ROMICAT) Study. Clin Chem 2011; 57: 1137-1145. 